\title{
A case of crush injury of the right foot in a 26-year-old man treated with platelet rich plasma and negative pressure
}

\author{
Paweł Zalita*, M.D.
}

\begin{abstract}
Treatment of a crush injury is a largely complicated and time-consuming process. Multidirectional damages often result in skin necrosis, oedema, blood circulation disorders and bone fractures. We present a case of a 26 year-old man with right foot crush injury. After trauma he underwent a complex therapy which embraced: anti-oedematous treatment, negative pressure wound therapy, reposition of the bones under X-ray control with the stabilization by means of Kirschner wires, immobilization and transdermal application of the platelet-rich plasma in the fracture fissure - as a nonunion treatment.
\end{abstract}

Keywords-NPWT, Endo-VAC, Endo-Sponge, anastomotic leakage, rectal cancer, postoperative complication

\section{INTRODUCTION}

C RUSH injury is a very serious and difficult to treat damage. It usually involves not only breakage of the soft tissue but also fracture of the osseous structures. One of the most serious complication associated with the crush injury is the compartment syndrome. It may lead to irreversible damages and high morbidity, therefore it is largely important to prevent this state or at least recognize it early. Treatment of the crush injury has to include early coverage of the skin loss, healing soft tissue damages and stabilization of the fractures. Unfortunately, despite early medical intervention some patients obtain poor treatment effects and suffer from serious complications. Vacuum therapy seems to be helpful in skin injury treatment, as well as using PRP in delayed bone healing.

\section{CASE Report}

A 26-year-old man was admitted to the Orthopedic Ward in The District Hospital in Poznan with the diagnosis of a crush injury of the right foot. After the accident the patient was transported by the ambulance to the Emergency Department (ED).

The physical examination revealed massive swelling of the right foot. The patient reported metatarsal pain on palpation, especially around the wound and above 3rd and 5th metatarsal bone. Linear wound length measured at the medial edge of the foot was about $15 \mathrm{~cm}$, while skin contusion at the dorsal side of the foot was $10 \mathrm{~cm}$ long and $8 \mathrm{~cm}$ wide. The pulse

Manuscript received 20.12.2014; revised 10.03.2015. No conflict of interest declared

Author affiliations: Department of Orthopedic and Traumatic Surgery of the District Hospital, Poznan., (PZ)

*Correspondence to: Paweł Zalita (e-mail: pawelzalita@interia.pl). on the posterior tibial artery and dorsalis pedis artery was palpable. Capillary refill time (CRT) was less than 2 seconds, temperature of the skin beyond the bruised area was regular, and the feeling in the foot was preserved. The patient did not report any chronic diseases. He was diagnosed with the fracture of the 3rd and the 5th metatarsal shafts according to the X-ray, accompanied by the wound and skin ulceration on the dorsal surface of the right foot (see figure 1).

Preliminary proceeding conducted in the Emergency Department included extensive washing with a saline solution and octenidine dihydrochloride. The wound was first anesthetized with $2 \%$ lidocaine, then provided with surgical sutures and finally covered with a sterile dressing. Due to the fact that the patient was recently vaccinated against tetanus, the next dose was not administered. The limb was immobilized in the cast and the patient was transferred to the Orthopedic Ward, where he received prophylactic antithrombotics: enoxaparin $40 \mathrm{mg} 1 \times 1$ s.c., pentoxifylline $2 \times 100 \mathrm{mg}$ i.v., rutin and aescelus hippocastanum extract $40,5 \mathrm{mg} 3 \times 2$ tabl., analgesics and wide-spectrum antibiotics - cefazolin $3 \times 1,0$ g i.m. and metronidazole $2 \times 500 \mathrm{mg}$ i.v.The dressings were changed two times a day using $3 \%$ boric acid compresses.

On the second day of hospitalization the patient exercised isometrically with a physiotherapist. During hospitalization the patient has developed skin necrosis corresponding to the foot ulceration area. After the reduction of massive oedema of the foot and emptying the content of ulcers, the patient was prepared for the surgery.

As the skin underwent necrosis, the negative pressuredressing called VAC (Vacuum-Assisted Closure) was applied to purify the wide area of skin necrosis at the dorsum side of the foot ${ }^{[2]}$ VAC was used for 4 days, the dressing was being changed once a day and the used pressure was around $-70 \mathrm{mmHg}$. It accelerated the process of epidermization of the wound. Then the standard compresses with octenidine dihydrochloride alternating with the dressings of 3\% boric acid were applied and at the very end paraffin dressings were also used ${ }^{3}$ Due to the high rate of swelling and deteriorating skin condition including the area of necrosis open reposition of the fractures under the control of the X-ray vision track, stabilization with Kirschner wires and evacuation of massive hematoma from metatarsus were done (see figure III).

The patient was discharged from the hospital on the third postoperative day after performing control $\mathrm{X}$-ray, to ensure that stabilizing material was in the proper position. The wound 


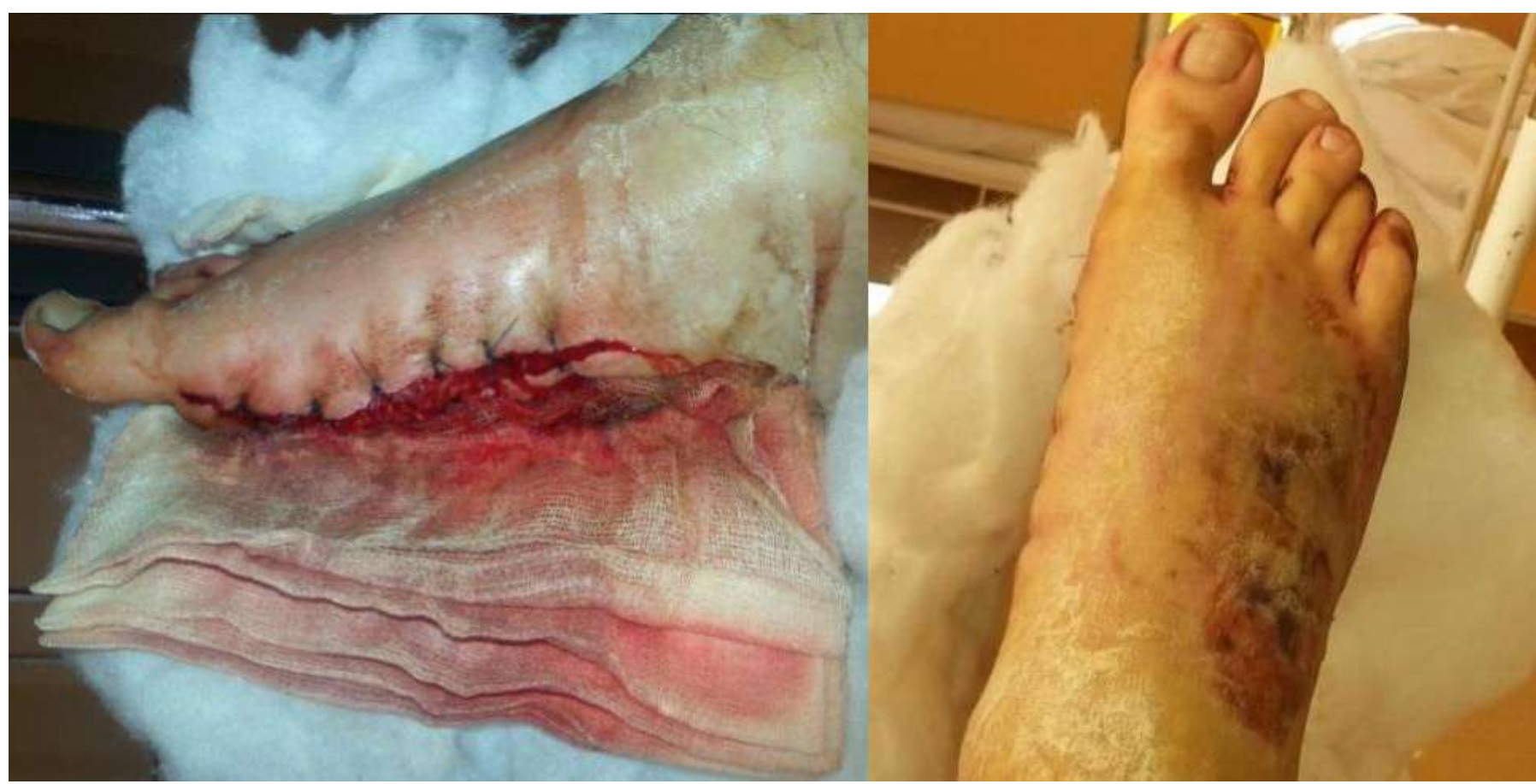

Figure 1. Clinical state of the patient 5 days after the injury. Left picture shows wound on the medial side of the right foot, while the picture on the right presents skin ulceration area on the dorsum of the foot.
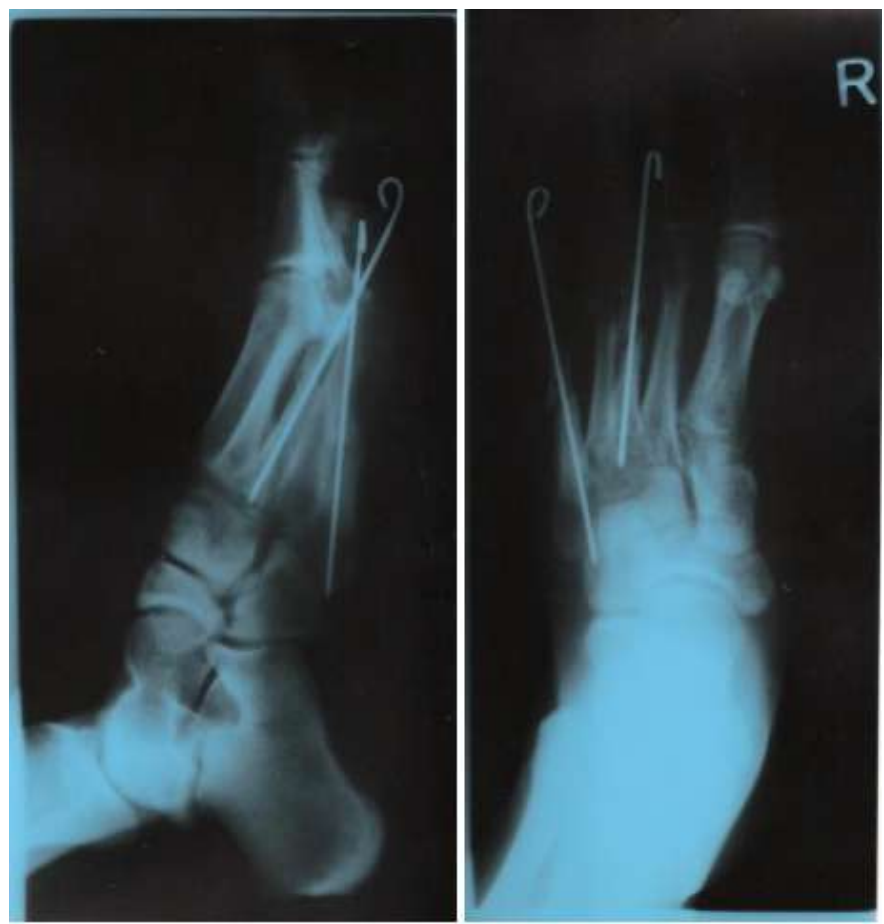

Figure 2. X-ray done 8 weeks after the surgery showing the nonunion in lateral projection (left figure) and anterio-posterior projection (right figure).

was almost healed, and the skin condition largely improved. There was a small area (10 $\mathrm{mm}$ long) of the wound secreting serum, but on the last day of hospitalization it was completely covered with granulation. The newly created granulation $(2.5$ $\mathrm{cm}$ long) was also noticed in the central part of the wound.
Operated limb was immobilized in a shank cast. The patient was advised to walk on elbow crutches and to spare the operated limb.

The dressings with a neomycin alternating with octenidine dihydrochloride were changed by the patient twice a day. $\mathrm{He}$ was also coming to control visits once a week for four weeks, until the area previously coated with the fresh granulation tissue was covered with normal skin.

The main difficulty in the treatment posed inappropriate bone healing. X-ray done six weeks after the surgery did not reveal callus in the fractures fissures. Therefore the immobilization was extended and ossein hydroxyapatite $2 \mathrm{x} 1$ in tabl. was administered. After next three weeks poor callus of the third metatarsal was observed in the X-ray. Unfortunately, fifth metatarsal bone did not respond to treatment. Three weeks later acceptable callus has appeared in the fracture fissure of the third metatarsal bone, therefore Kirschner wire was removed. The physical examination brought out pain on palpation and pathological fracture mobility of the fifth metatarsal indicating nonunion confirmed by the X-ray.

The Kirschner wire was removed from the fifth metatarsal and the patient was allowed to walk without additional medical equipment. At the same time the patient was qualified to the transdermal application of platelet-rich plasma in the fracture fissure 5 . 10 Sixteen weeks after the surgery the patient was admitted to the hospital for the introduction of plateletrich plasma (PRP) in the fracture gap by the mean of GPS Biomet under the control of the X-ray vision. ${ }^{6}$ PRP was prepared according to manufacturer's instructions. $54 \mathrm{ml}$ of the patient's blood was collected to the test-tube containing citrate. Then the test-tube was placed in the separator, which enabled the identification of the individual cellular components 


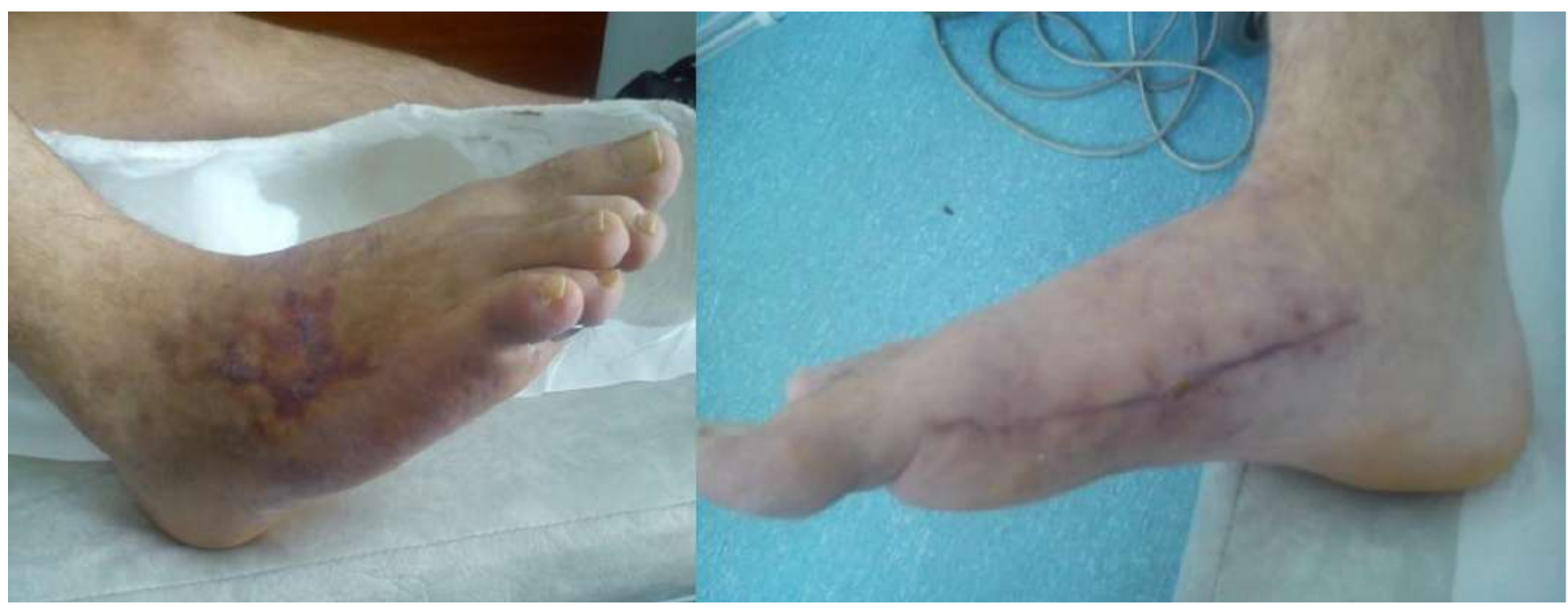

Figure 3. Patient's foot 7 weeks after the injury. Pictures are showing new epidermis on the dorsum of the foot (left) and the scar on the medial side of the foot (right).

of blood, platelet-rich plasma and platelet-poor plasma. The blood of the patient was centrifuged for 15 minutes at 3200 turnover/minute. Finally, $5 \mathrm{ml}$ of the platelet rich plasma carrying a growth factors (PDGF, TGF, $\beta 1, \beta 2$, VEGF, EGF, IGF-1, fibronectin, fibrin and vitronectin) were obtained 8 According to the manufacturer, it is possible to increase concentration of the platelets even 9 times, which provides a higher concentration of the growth factors accelerating osteosynthesis. Nonsteroidal anti-inflammatory drugs (NSAIDs) administration was abandoned in order not to inhibit platelet degranulation. The patient left the hospital few hours after the surgery.

After four weeks the patient appeared for the follow up. There was no pathological mobility of bone fragments or pain of the fifth metatarsal, X-ray images taken in two projections revealed extensive callus. The patient was very satisfied with the treatment result and made almost a full recovery. The only dysfunction is a slightly limited range of motion of the toe, resulting from the extensive cicatrices located on the course of tendons of the extensor muscle (see figure 3 ).

\section{DISCUSSION}

Crush injuries always involve damages of soft tissues and bones. These injuries are very serious and pose significant amount of clinical problems. To achieve progress in healing and avoid severe complications, applied treatment must be multidirectional. It seems like a key to success is to conduct not only antioedematous treatment, debridement of necrotic tissue and skeletal stabilization, but also try to avoid secondary soft tissue damages and complications.

The use of platelet-rich plasma in the treatment of delayed union of bones seems to be an excellent method, but it has some constraints. ${ }^{4}$ It has to be limited to the group of patients characterized by very good healing potential. In our case one transdermal application of PRP allowed for achievement of remarkable therapeutic effect, but Seijas et al! described $^{8}$ the case, in which three percutaneous injections of PRP in delayed union of the clavicle finally enabled the union after three months. This method does not replace surgery, but it can be added to the standard treatment. Bielecki et al. . $^{1}$ described the case, in which transdermal application of PRP enabled union of the fracture in 13 out of 20 patients. Although PRP application still requires further research, it seems to be an interesting method of treatment which might accelerate desired therapeutic effect.

\section{CONCLUSION}

Transdermal application of the Platelet-Rich Plasma in delayed union and in selected cases of nonunion is a safe and promising method of faster bone healing treatment, ${ }^{7}$ while Negative Pressure Wound Therapy (NPWT) becomes a standard therapy of chronic wounds with impaired healing. It improves rate of epidermization, protects the wound from infections and helps to decrease the exudate from the wound which is very often not possible by the mean of conventional dressings. It can also be used in a variety of impaired wound healing disorders and reduces time of patient's hospitalization.

\section{REFERENCES}

[1] T. Bielecki, T.S. Gazdzik, and T. Szczepanski. Benefit of percutaneous injection of autologous platelet-leukocyte-rich gel in patients with delayed union and nonunion. European Surgical Research, 2008. URL: http://dx.doi.org/10.1159/000114967

[2] M. Diefenbeck, U. Mennenga, P. Gückel, A. Tiemann, T. Mückley, and G. Hofmann. Vakuumtherapie bei haut- und weichgewebsinfektionen der extremitäten. nutzen des wundabstrichs bei der planung des sekundären wundverschlusses? Zeitschrift für Orthopädie und Unfallchirurgie, 2011. URL: http://dx.doi.org/10.1055/s-0030-1250694

[3] L Huang, F Zhang, PH Ye, XF He, YZ Zhu, and YP Ruan. Application of vacuum sealing drainage in open ankle fracture and dislocation. Zhongguo Gu Shang, 2012.

[4] S.R. Kanthan, G. Kavitha, S. Addi, D.S.K. Choon, and T. Kamarul. Platelet-rich plasma (PRP) enhances bone healing in non-united criticalsized defects: A preliminary study involving rabbit models. Injury, 2011. URL: http://dx.doi.org/10.1016/j.injury.2011.01.015

[5] Meir Liebergall, Josh Schroeder, Rami Mosheiff, Zulma Gazit, Zilberman Yoram, Linda Rasooly, Anat Daskal, Amal Khoury, Yoram Weil, and Shaul Beyth. Stem cell-based therapy for prevention of delayed fracture union: A randomized and prospective preliminary study. Mol Ther, 2013. URL: http://dx.doi.org/10.1038/mt.2013.109 
[6] Massimo Mariconda, Francesco Cozzolino, Andrea Cozzolino, Elio D’Agostino, Antonio Bove, and Carlo Milano. Platelet gel supplementation in long bone nonunions treated by external fixation. Journal of Orthopaedic Trauma, 2008. URL: http://dx.doi.org/10.1097/BOT. 0b013e318172cea5

[7] Yoichiro Ogino, Yasunori Ayukawa, Toshio Kukita, and Kiyoshi Koyano. The contribution of platelet-derived growth factor, transforming growth factor- $\beta 1$, and insulin-like growth factor-i in platelet-rich plasma to the proliferation of osteoblast-like cells. Oral Surgery, Oral Medicine, Oral Pathology, Oral Radiology, and Endodontology, 2006. URL: http://dx. doi.org/10.1016/j.tripleo.2005.08.016

[8] R Seijas, RY Santana-Suarez, M Garcia-Balletbo, X Cuscó, O Ares, and $\mathrm{R}$ Cugat. Delayed union of the clavicle treated with plasma rich in growth factors. Acta Orthop Belg, 2010.

[9] A. H. R. W. Simpson. The role of growth factors and related agents in accelerating fracture healing. Journal of Bone and Joint Surgery - British Volume, 2006. URL: http://dx.doi.org/10.1302/0301-620X.88B6.17524

[10] AHR Simpson, L Mills, and B Noble. The role of growth factors and related agents in accelerating fracture healing. International Bone and Joint Surgery.

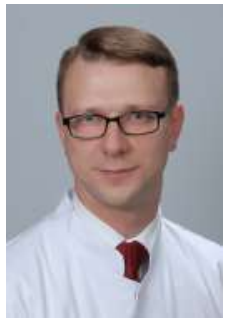

Paweł Zalita, M.D. is an assistant physician at the Orthopedic Ward at the District Hospital in Poznan. $\mathrm{He}$ is particularly interested in the treatment of sport injuries and arthroscopic surgery. He also is a football fan and a jogger. 